\title{
Dipole moments and melting points and their unsolved miracles on the application of hammett equation
}

\author{
R. Sanjeev ${ }^{1}$, V. Jagannadham ${ }^{2} *$, R. Veda Vrath $^{3}$ \\ ${ }^{1}$ Departments of Chemistry, Mizan-Tepi University, Tepi Campus, Tepi, Ethiopia \\ ${ }^{2}$ Departments of Chemistry, Osmania University, Hyderabad, India \\ ${ }^{3}$ Departments of Chemistry, L N Gupta Evening College, Hyderabad,India
}

Email address:

jagannadham1950@yahoo.com (V. Jagannadham)

To cite this article:

R. Sanjeev, V. Jagannadham, R. Veda Vrath. Dipole Moments and Melting Points and Their Unsolved Miracles on the Application of Hammett Equation. Education Journal. Vol. 1, No. 1, 2012, pp. 1-4. doi: 10.11648/j.edu.20120101.11

\begin{abstract}
Application of Hammett equation to structure-reactivity correlations of a "localized reaction site" in elucidating the reaction mechanisms of several organic reactions is a very well-knownphenomenon in physical-organic chemistry class-room of senior undergraduate and graduate level students. This is a testament of purely chemical phenomena. There were twosuccessful reports in literature on the application of linear and non-linearHammett equation to claim to be physical property of solubilities of benzoic acids. In this article we tried to apply the same to the dipole moments and melting points of some benzoic acidsand however premature andunsolved.
\end{abstract}

Keywords: Hammett Equation, Melting Points Of Benzoic Acids, Dipole Moments Of Benzoic Acids, StructureReactivity Correlations

\section{Introduction}

Quantitative solubility-structure relationships for severalmeta- and para-substituted benzoic acids in benzene and in cyclohexane1 and in 1, 4-dioxane and tetrahydrofuran (THF)2 were studied. Even the application of Hammett equation to melting points of some benzene derivatives were touched and left without any reasonable arguments3.Katritzky et al gave a one line concluding argument on the application of QSPR models to the melting points of benzene derivatives in terms of molecular packing and intermolecular interactions4.The V-shaped locus in the Hammett plot was not noticed in the study of effect of substituents on dipole moments of benzoic acids5. In this the non-linearity is concluded in terms of poor correlation, hence no arguments were offered. In the present article we were tempted to apply the Hammett equation to the dipole moment and melting point data of benzoic acids but still it kept us under skepticism.

\section{Discussion}

Though lately the application of structure-reactivity correlations in the form of either linear or non-linear Hammett equation isnot being observed in chemical literature, there are many still unsolved applications in this aspect with several physical properties such as dipole moments, melting points, surface tension, viscosities and several other physical properties as a function of Hammett substituent constants.Our recent experience in this field ${ }^{6-12}$ and in the interpretation of Marcus equation (a special type of linear free energy relationship) to electron transfer reactionsbetween carbon centered free radicals and aromatic systems ${ }^{13}$, prompted us to take up the title subject and if possible to offer any reasonably intelligent explanations. In the study of quantitative solubility-structure relationships for several meta- and para- substituted benzoic acids in benzene and in cyclohexane ${ }^{1}$, Hancock et al observed that there was no significant correlation between $\log \mathrm{X}_{\mathrm{B}}$ alone or $\log \mathrm{X}_{\mathrm{C}}$ alone with Hammett's $\sigma$. Here $\mathrm{X}_{\mathrm{B}}$ and $\mathrm{X}_{\mathrm{C}}$ are the solubilities of benzoic acids in benzene and cyclohexane. However they observed a linear correlation between $\log \left(\mathrm{X}_{\mathrm{B}} / \mathrm{X}_{\mathrm{C}}\right)$ and Hammett's $\sigma$. They explained this as a measure of tendency of the interaction of $\pi$-electron cloud of benzene with the benzoic acid. And the same studies by these people in 1,4dioxane and THF were performed ${ }^{2}$ and they have observed a V-shaped locus when $\log \left(\mathrm{X}_{\mathrm{D}} / \mathrm{X}_{\mathrm{C}}\right)$ were plotted against $\sigma$. Here $X_{D}$ is the solubility of benzoic acids in dioxane and $\mathrm{X}_{\mathrm{C}}$ is from reference 1 . Hancock et al had tried to explain this V-shaped Hammett locus based on the same explana- 
tions of change in mechanisms of Wolf-Kishner reaction of hydrazones ${ }^{14}$ and U-shaped Hammett locus for the rates of solvolysis and thiosulfate reactions of $\alpha$-chloro toluenes ${ }^{15}$ for two sets of electron donating and electron withdrawing of substituents. But it was difficult to make any reasonable explanations due to complexity of several factors which affect the solution processes. Later Schreck ${ }^{16}$ had described this in an article on non-linear Hammett relationships as what appears to be the only physical property that gives a non-linear Hammett plots. But these are not completely physical properties as the solubility would be a function of ionizing capacity and ionization is a chemical property which depends on the dielectricity of the solvent and it will be taking place at alocalized ionization site.This gets support from the variation of Hammett $\rho$ with solvent polarity $^{17,18}$.

\section{Dipole Moments}

To our view it may not be unreasonable to assume that the dipole moments and melting points to be the purely physical properties. The dipole moment is a property of a molecule that results from charge separation with in a bond of a diatomic molecule containing two hetero atoms. The larger the difference in electronegativities of the two hetero atoms the greater would be the dipole moment. However in polyatomic molecules it is not possible to measure the dipole moment of an individual bond with in a molecule, but one can measure only the total moment of the molecule, which is the vectorial sum of the individual bond mo- ments ${ }^{19}$. In all the molecules these individual moments are constant but may not be taken as universal ${ }^{20}$. Therefore one can conclude that the property of the molecule in terms of dipole moment is not like a localized reaction center but it is the bulk property of the entire molecule. The difference between electroneagativities of carbon and hydrogen is so small, alkanes or any hydrocarbon molecule had very small dipole moment. For example the dipole moment of benzene is zero ${ }^{21}$.If we introduce a substituent with or without hetero atom in to the benzene ring the dipole moment would be > $0^{20}$. This is because of the charge separation analogous to resonance in the entire aromatic molecule. Therefore whether the substituent is either electron donating or electron withdrawing the dipole moments of mono substituted benzenes ought to be more than that of benzene because either of the substituents would induce charge separation in the benzene ring. As an example ${ }^{22}$, the dipole moment of toluene is $0.43 \mathrm{D}$ and that of nitrobenzene is $3.93 \mathrm{D}$. It is a general observation that when a substituent introduced in to benzene ring, having hetero atom other than carbon or hydrogen the dipole moment would be even large. The dipole moment of benzoic acid is again $1.78 \mathrm{D}^{5}$. Hence the dipole moments of substituted benzoic acids either with electron donating or electron withdrawing substituents would be more than that of benzoic acid itself ${ }^{5}$. Surprisingly there was a good correlation between dipole moments of benzoic acids with Hammett substituent constants with two straight lines one with negative slope for electron donating substituents and the other with positive slope for electron with drawing substituents (Figure 1).

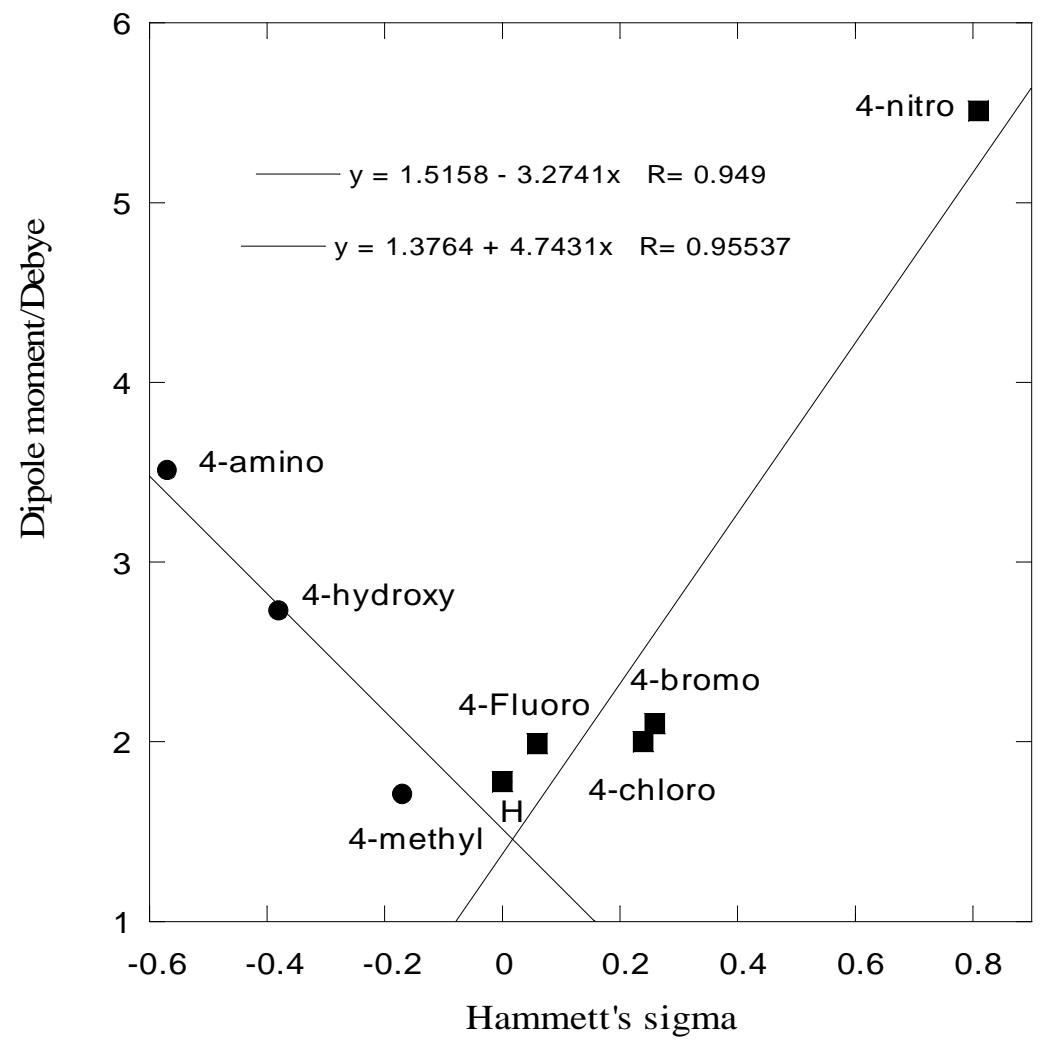

Figure 1. Hammett plot: dipole moments of benzoic acids versus Hammett's sigma. 
The dipole moments of different benzoic acids are from references 5 and 20. Though the correlation coefficients are bit away from unity, the trends are unmistakable. Therefore the dipole moments are fairly good indications of application of the Hammett equation. We claim this is the first observation on the application of Hammett equation to a physical property like dipole moments.

\section{Melting points}

A century ago Lindemann had put forward a theoretical basis to predict the bulk melting point of crystalline materials ${ }^{23}$. According to his theory, the average amplitude of thermal vibrations increases with increase in temperature. Melting of the crystalline solidwould start when the amplitude of vibration becomes large enough for adjacent atoms to partly occupy the same space. The Lindemannprediction states that melting is expected when the root mean square vibration amplitude exceeds a threshold value. According to equipartition theorem ${ }^{24}$ assuming all atoms in crystalline substance vibrate with same frequency, $v$, the estimated average thermal energy was given by the following equation.

$$
\mathrm{E}=4 \pi^{2} m v^{2} \mathrm{u}^{2}=k_{\mathrm{B}} \mathrm{T}
$$

Where $\mathrm{m}$ is the atomic mass, $v$ is the frequency, uis the average vibration amplitude, $k_{\mathrm{B}}$ is the Boltzmann constant, and $\mathrm{T}$ is the absolute temperature.If the threshold value of $\mathrm{u}^{2}$ is replaced by $\mathrm{c}^{2} \mathrm{a}^{2}$ where ' $\mathrm{c}$ ' is the Lindemann constant and ' $a$ ' is the atomic spacing, then the equation for melting point is given by:

$$
\mathrm{T}_{\mathrm{m}}=\frac{4 \pi^{2} \mathrm{~m} v^{2} \mathrm{c}^{2} \mathrm{a}^{2}}{k_{\mathrm{B}}}
$$

On right hand side of equation 2 only the quantity ' $\mathrm{m}$ ' is variable assuming the atomic spacing ' $a$ ' in a molecule to be constant and as others are all constants. Therefore as molecular mass of a molecule consisting of several atoms increases the average thermal energy required for a crystalline substance to melt increases to break more number of bonds. Hence among the all the substituted benzoic acids, un-substituted benzoic acid would have a lower melting point and other substituted benzoic acids whether they had electron donating or electron withdrawing substituent would have higher melting points. Again surprisingly there was a good correlation between melting points of benzoic acids with Hammett substituent constants with two straight lines one with negative slope for electron donating substituents and the other with positive slope for electron withdrawing substituents (Figure 2).

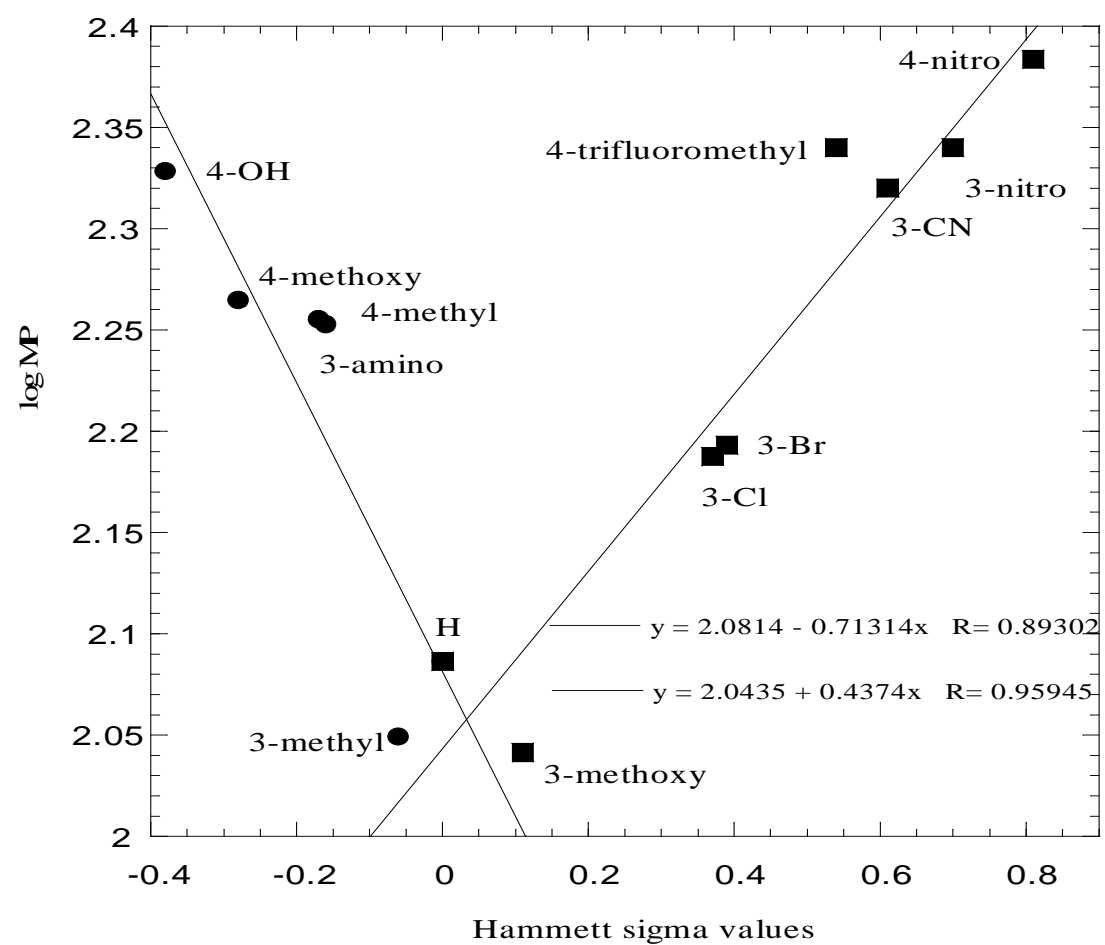

Figure 2. Hammett plot of log (melting point) of benzoic acids versus Hammett substituent constant.

The melting points used in this Hammett correlation are from 'Google search Engine' using the molecular formula of each benzoic acid.

It has to be realized that the original Lindemann's model for vibrational melting, like many of hissuccessor Scientists, refers only to a crystal with the simple structure. Crystals containing big molecules exhibit complex vibrations. This may rule out any simple rule of lattice stability, determined 
merely by vibrational amplitudes of the molecular centers of mass. Furthermore, the Lindemann model is based on harmonic forces whereas melting must involve bond breaking. This is another serious defect of the model. The other defect of the model is that melting is described in terms of individual atomic property, i.e. mean square amplitude of vibration, while a phase transition is a cooperative bulk process. In addition, the Lindemann model did not include the idea of the melting transition must involve both solid and liquid phases. Nevertheless the predictive success of the Lindemann melting criterion lent support to the belief that melting could be a gradual process, beginning within the solid at temperatures below the melting point. With these defects in Lindemann's theory, it may be premature to apply the Hammett equation to the melting point data but we have given a trial for another physical property of melting point.

\section{References}

[1] C. Kenny Hancock, John N Pawloski and John P. Idoux, Quantitative solubility-structure relationships for some meta- and para-substituted benzoic acids in benzene and in cyclohexane, J. Org. Chem., (1966) 31, 3801.

[2] C. Kenny Hancock and John P. Idoux, Quantitative solubility-structure relationships for some meta- and parasubstituted benzoic acids in 1, 4-dioxane and in tetrahydrofuran, J. Org. Chem., (1967) 32, 1931.

[3] M. Ludwig, S. Wold and O. Exner, The role of meta and para-benzene derivatives in the evaluation of substituent effects: a multivariate data analysis, Acta. Chem. Scand., (1992) 46, 549

[4] M. Karelson, U. Maran, Y. Wang and A. R. Katritzky, QSPR and QSAR models derived using large molecular descriptor spaes,www.ark.chem.ufl.edu/Published_Papers/PDF/744.pd f.

[5] F. P. Parungo and J. P. Lodge Jr., Molecular structure and ice nucleation of some organics, J. Atmospheric Sciences, (1965) 22,309 .

[6] V. Jagannadham and R. Sanjeev, Effect of Hetero Atom on the Hammett's Reaction Constant ( $\rho$ ) from the Physical Basis of Dissociation Equilibriums of (dithio) Benzoic Acids and (thio) Phenols and its Application to Solvolysis Reactions, Advances in Physical Chemistry, published by Hindawi publishers, USA, Volume 2012 (2012), Article ID 598243, 1-4 pages.

[7] V. Jagannadham, R. Sanjeev and R. Veda Vrath, Hammett equation - A tool for the identification of the site of attack in aromatic systems: One hour classroom lecture for physical organic chemistry graduate students, ChemEdNZ, (New Zeeland Journal of Chemical Education) (2012) November, page 14.

[8] R. Sanjeev, V. Jagannadham, R. Veda Vrath, A simple explanation from the sign of $\rho$-value for the kind of charge that develops in the intermediate or the transition state of a reaction series: A one-hour physical-organic chemistry graduate classroom lecture, Khimiya/Chemistry (The Bulgarian Journal of Chemical Education), (2012) Vol. 21, page $71-77$.

[9] R. Sanjeev and V. Jagannadham,Effect of hybridization of carbon on Hammett reaction constant $(\rho)$ : $\pi$-electron transmission versus $\sigma$-electron insulation: A motivating lecture for graduate students of physical-organic chemistry classroom, American Journal of Organic Chemistry, Published by Scientific and Academic Publishing, USA, (2012) vol. 1 (in press).

[10] V. Jagannadham, Linear free energy relationships (LFER) as a one hour class-room lecture for post-graduate students: Correlation of the nature of the transition states. Chemical Education Journal, Japan, (2009), Vol. 12, No. 1. http://chem.sci.utsunomiya-u.ac.jp/cejrnlE.html.

[11] V. Jagannadham, The attenuation effect through methylene group. Bulgarian Chem. Commns., (2009) vol. 41, page 50.

[12] V. Jagannadham and R. Sanjeev, the Marvelous Marcus equation: Distinguishing inner-sphere electron transfer reactions from outer-sphere electron transfer reactions: A one hour graduate class-room lecture, Bulgarian Chemical Communications, (2011) vol. 42, page 383-394.

[13] H. H. Szmant and C. Harmuth, the Wolff-Kishner Reaction of Hydrazones, J. Am. Chem. Soc., (1964) 86, 2909.

[14] R. Fucks and D. M. Carltoo, Substituent Effects in the Solvolysis and Thiosulfate Reactions of 3-, 4- and 3,5Substituted $\alpha$-Chlorotoluenes, J. Am. Chem. Soc., (1963) 85, 104.

[15] J. O. Schreck, Non-Linear Hammett relationships, J. Chem. Edn. (1971) 48, 103

[16] C. D. Ritchie and R. E. Uschold, Acidity in non-aqueous solvents. VI. Further studies of weak acids in dimethyl sulfoxide solution, J. Am. Chem. Soc., (1968) 90, 2821.

[17] I. M. Kolthoff and M. K. Chatooni, Substituent effects on dissociation of benzoic acids and heteroconjugation of benzoates with p-bromophenol in acetonitrile, N,Ndimethylformamide, and dimethyl sulfoxide. Intramolecular hydrogen bonding in o-hydroxybenzoic acids and their anions, J. Am., Chem. Soc., (1971) 93, 3843.

[18] O. Exner, Dipole moments in organic chemistry, Georg Theime Publishers, Stuttgart, 1975.

[19] O. Exner, Collection of dipole moments in Collect. Czech. Chem.Commun. (1985) 50, 188 and 200.

[20] Benzene- Wikipedia, the free encyclopediaen.wikipedia.org/ wiki/Benzene.

[21] J. March, Advanced Organic Chemistry: Reactions, mechanisms and structure, Wiley Inter-science publication, John Wiley \& Sons, 1992, page 16 and references cited there in.

[22] F. A. Lindemann, "The calculation of molecular vibration frequencies". Physik. Z. (1910) 11, 609-612.

[23] S. Sorkin, Point defects, lattice structure, and melting, Thesis, Technion, Israel (2003). 